\title{
Wavenumber-frequency analysis of river surface texture to improve accuracy of image-based velocimetry
}

\author{
Kojiro Tani ${ }^{1, *}$ and Ichiro Fujita ${ }^{1}$ \\ ${ }^{1}$ Graduate School of Engineering, Kobe University, 657-8501 1-1 Rokkodai, Nada, Kobe, Japan \\ ${ }^{2}$ Department of Civil Engineering, Kobe University, 657-8501 1-1 Rokkodai, Nada, Kobe, Japan
}

\begin{abstract}
In the unseeded image-based techniques for river surface flow measurements, advection speed of surface textures composed of surface ripples or floating objects is measured by image analysis. However, the methods would yield erroneous information when the surface texture is affected by gravity waves propagating in all directions. In order to improve the measurement accuracy, such wave effects have to be subtracted in the image analysis. For that purpose, a wavenumber-frequency analysis was applied to a space-time image (STI) generated in the space-time image velocimetry (STIV) analysis and succeeded in eliminating the wavegenerated pattern contained in the texture in STI. It was made clear that turbulence-generated texture propagates at the speed of surface flow.
\end{abstract}

\section{Introduction}

In the past decades, image-based surface velocity measurement techniques, such as the Large Scale Particle Image Velocimetry (LSPIV) [1] and Space-Time Image Velocimetry (STIV) [2] have been paid attention and utilized to measure river surface velocity. These two techniques are also applied to airborne images shot from a drone or a helicopter and they were successfully used to measure flood flows over a wide region. These image-based velocimetries assume that the river surface textures caused by the free-surface fluctuations travel with the surface flow. However, the assumption might be failed when the effects of gravity on the free-surface becomes dominant rather than the effect of sub-surface turbulence because gravity waves propagate in all directions. Therefore, in order to improve accuracy and robustness of image-based velocimetries, it is necessary to remove the effect of gravity waves.

In laboratory scale studies, there are some researches focusing on free surface behaviour in open-channel flows [3-6], in which the free surface is considered to be affected by either sub-surface turbulence or capillary-gravity waves depending on the Froude number or the bed roughness condition. However, the dependency of parameters is not clearly investigated in the past researches. Moreover, water surface behaviour in river flows is even more difficult to investigate because of the difficulty of measuring free surface profiles in the field. Therefore, instead of physically measuring water surface profiles, river surface video images shot from a river bank was used by assuming that the dark and bright portions corresponds to the concave and convex portions of water surface, respectively. In this research, the 
wavenumber-frequency analysis [5,6] was applied to the surface images to isolate the wave effect.

\section{Outline of field measurement}

The river surface images were obtained during the field measurement campaign conducted at a site of the Shinano river in Niigata prefecture, Japan from April 26 to 28 . The total study area was upstream and downstream of the Asahi Bridge and seven locations were prepared for image shooting as shown in Figure 1. The video images were captured at each location using a camcorder (Sony FDR AXP35) with $4 \mathrm{~K}$ resolution at a sampling rate of $30 \mathrm{~Hz}$. The water level was measured by five water gauges as shown in Figure 1. In this paper, we pay attention to the video images shot from Camera \#5. The average water depth was about five meters but a little deeper nearer to the left bank.

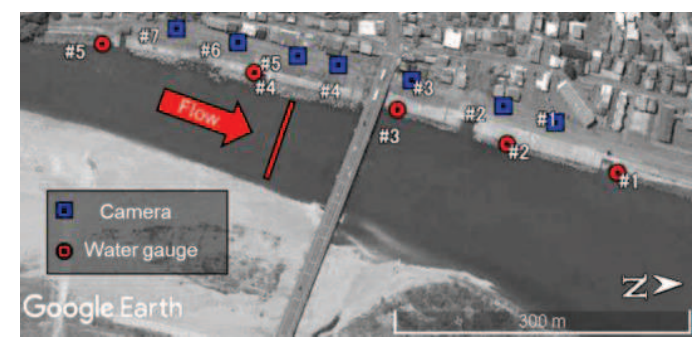

Fig. 1. Overview of study area

\section{Image processing method}

\subsection{Geometric rectification of image}

The surface video images were shot obliquely from the left bank as indicated in Figure 1. Therefore, it is necessary to ortho-rectify the oblique images for the image analysis. The image transformation was simplified by capturing images using a tripod with a high-accurate clinometer. With this setup, the image transformation from the physical coordinates $(x, y, z)$ to the image coordinates $(u, v)$ can be conducted by using the angle of repose $\rho$, the focal length $f$, and the vertical distance from water level to the camera lens $d$, as shown in eq.(1) and eq.(2).

$$
\begin{gathered}
u=-f \frac{x \cos \rho+y \sin \rho}{z-d} \\
v=-f \frac{-x \sin \rho+y \cos \rho}{z-d}
\end{gathered}
$$

In this study, the angle $\rho$ was measured accurately by the clinometer and $d$ was measured by a laser range finder. This instrument makes it much easier to generate rectified images than the conventional method that requires more than six ground control points. As an example, original and rectified images are shown in Figure 2.

\subsection{Space-Time Image}

In order to extract the free surface behaviour from the video images, space-time images (STIs) were created for a search line set in the streamwise direction to use in STIV analysis. 




(a) original image

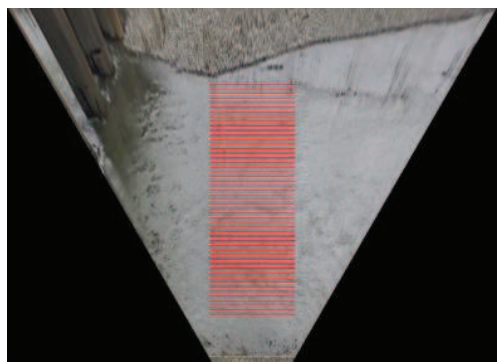

(b) ortho-rectified image

Fig. 2. Example of geometric rectify

In an STI, time evolution of surface textures affected by subsurface turbulence and gravity waves are included as will be shown later. If we assume that the surface texture is advected at the same speed of surface flow, the gradient of the texture appeared in an STI should be directly related to the surface flow velocity. STIV is a technique to measure such texture gradient efficiently. However, STI sometime contains unevenly distributed image intensity which can become a source of error. Therefore, a standardization filter expressed by eq.(3) is first applied to an STI.

$$
I^{S}(x, t)=\left(I(x, t)-\mu_{t}(x)\right) / \sigma_{t}(x)
$$

Here, $I(x, t)$ and $I^{S}(x, t)$ are the original and filtered images respectively, $\mu_{t}$ is the mean value for each vertical array and $\sigma_{t}$ is its standard deviation. This filter enhances the variation of image intensity within an STI uniformly. It was confirmed that the fluctuation of image intensity after applying the filter follows the Gaussian distribution, which is the same feature of water surface fluctuation measured by a sophisticated surface measurement technique [6].

\section{Results and discussions}

\subsection{Space-time image}

In the present analysis, fifty standardized STIs are created from the video shot from the Camera \#5. The search lines are set at a constant spacing of $1.76 \mathrm{~m}$ with a streamwise length of $37 \mathrm{~m}$, as indicated by red lines in Figure 2. Regarding the vertical time scale, 20 seconds of images that correspond to 600 frames were used. Figure 3 shows some examples of standardized STIs. Although there appears various textures in STIs, the major inclined pattern can be visually identified which expresses the phase velocity of the surface fluctuations. However, in addition to the above overall pattern, textures having different oscillatory patterns can also be seen in each STI. The former linear texture is considered to be generated by the subsurface turbulence travelling with the surface flow. On the other hand, the latter additional textures are considered to be caused by the free surface waves. This fact agrees with some past studies in the laboratory [6].

\subsection{Wavenumber-freqency spectrum}

To examine the texture features of STIs in frequency domain, the wavenumber-frequency spectrum was calculated by applying the two-dimensional discretised Fourier transformation (DFT) to a standardized STI as follows.

$$
S^{S}\left(k_{x}, \sigma\right)=\frac{1}{4 \pi^{2}} \iint\left\langle I^{N}(x, t) I^{N}(x+\rho, t+\tau)\right\rangle e^{-i\left(k_{x} \rho+\sigma \tau\right)} d \rho d \tau
$$


Here, $k_{x}$ and $\sigma$ are streamwise wavenumber and angular frequency, respectively. Figure 4 shows some examples of wavenumber-frequency spectrum, calculated from STIs shown in Figure 3. The plus signs shown over the colour plots exhibit the location of the local maxima, estimated by comparing surrounding eight points. The regions with high energy appear trigeminal from near the origin toward the first quadrant.

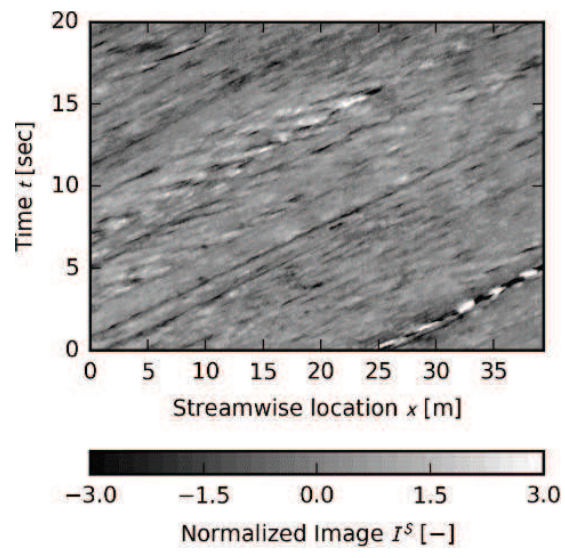

(a) $z=35.3 \mathrm{~m}, h=4.94 \mathrm{~m}$

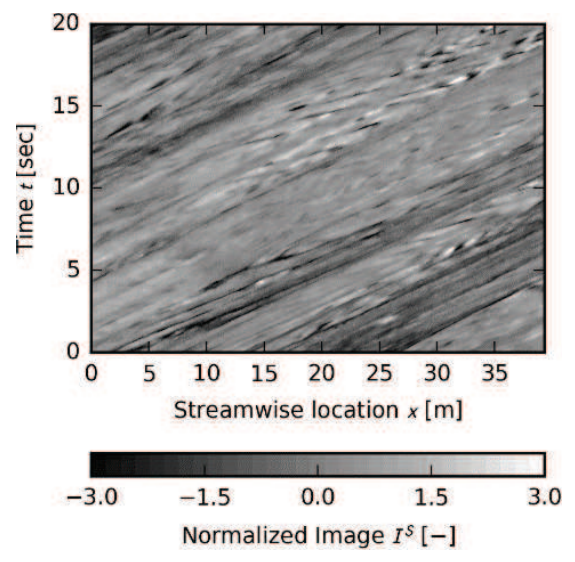

(b) $z=70.6 \mathrm{~m}, h=3.35 \mathrm{~m}$

Fig. 3. Standardized STIs ( $z$; distance from the left bank)

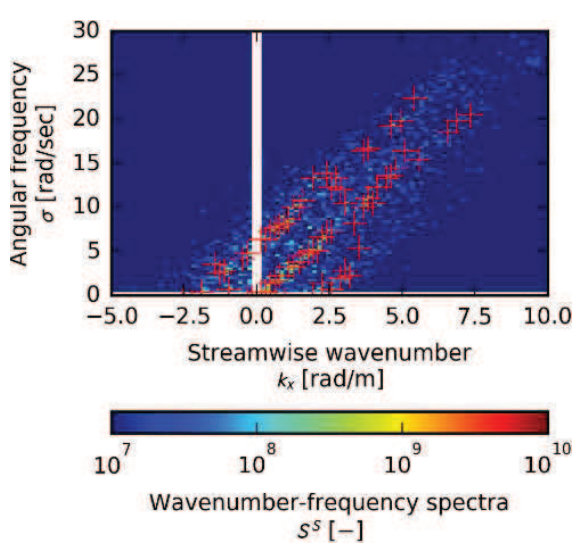

(a) $z=35.3 \mathrm{~m}, h=4.94 \mathrm{~m}$

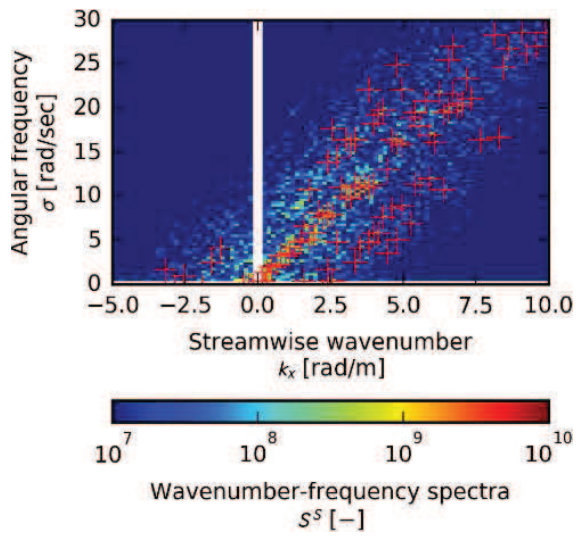

(b) $z=70.6 \mathrm{~m}, h=3.35 \mathrm{~m}$

Fig. 4. Wavenumber-frequency spectra of standardized STIs

Comparing the results with those obtained in the laboratory experiments $[5,6]$, the tips of these high energy regions seem to correspond to the region expressed by the dispersion relationships of turbulence and gravity waves. The dispersion relationship of the frozen turbulence is given as follows.

$$
c=\sigma / k_{x}=u_{S}
$$

Here, $c$ is the phase velocity and $u_{s}$ is the mean surface velocity. Considering the Doppler shift from the mean velocity, the dispersion relationship of gravity waves is described by the micro amplitude wave theory as follows. 


$$
c=u_{s} \pm\left\{g / k_{x} \cdot \tanh \left(k_{x} h\right)\right\}^{0.5}
$$

Here, $g$ is the gravitational acceleration and $h$ is the mean water depth. Considering the gravity wave propagates in the all directions, plus and the minus signs correspond to advancing and receding of the gravity waves. When the water depth is deep enough, the effect of the underwater becomes negligible and the wavelength does not depend on the water depth. Such waves are called "deep water waves" and the dispersion relationship is given as follows.

$$
c=u_{s} \pm\left(g / k_{x}\right)^{0.5}
$$

Considering the gravity waves propagate in all directions, the mean surface velocity can be estimated from the local maxima locations by the least square method.

$$
u_{s}=\sum \sigma^{P} k_{x}^{P} / \sum k_{x}^{P^{2}}
$$

Here, a variable with the superscript $P$ stands for the location of local maxima. Figure 5 shows the dispersion relationship induced from the local maxima of the spectra. The three curves indicated in the figure show the dispersion relationships described by eq. (8), (9) and (10). The dispersion relationships extracted from the STIs show good agreement with the theories of both of turbulence and waves, excluding the region near the wavenumber close to zero. This disagreement is considered to be just caused by the lack of the resolution and not so important.



(c) $Z=35.3 \mathrm{~m}, h=4.94 \mathrm{~m}$

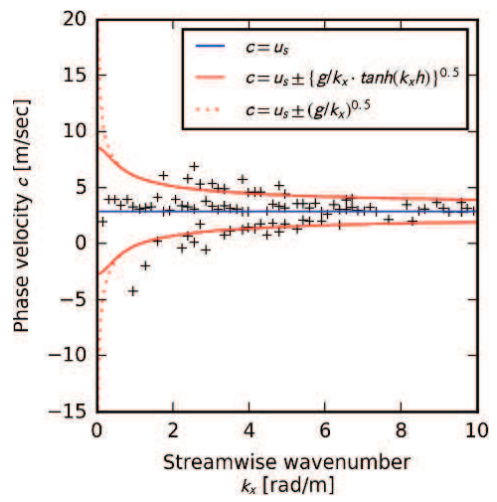

(d) $z=70.6 \mathrm{~m}, h=3.35 \mathrm{~m}$

Fig. 5. Dispersion relationships extracted from STIs

Figure 6 shows the dispersion relationships extracted from all of the STIs. Since the shape of the curves described by eqs. (5-7) should change depending on the water depth, the respective relations are non-dimensionalized as follows.

$$
\hat{k}_{x}=k_{x} h, \hat{\sigma}=\sigma h /(g h)^{0.5}
$$

As the results of the nondimensionalization, eq. (9), eq. (10) and eq. (10) are rewritten as follows respectively.

$$
\begin{gathered}
\hat{c}=F_{S} \\
\hat{c}=F_{S} \pm\left\{\hat{k}_{x} \cdot \operatorname{coth}\left(\hat{k}_{x}\right)\right\}^{-0.5} \\
\hat{c}=F_{S} \pm \hat{k}_{x}^{-0.5}
\end{gathered}
$$

Here, $F_{S}$ is the Froude number using the mean surface velocity as a representative velocity, defined by

$$
F_{s}=u_{s} /(g h)^{0.5}
$$


The curves in Figure 6 correspond to those indicated in Figure 5. It is obvious that the measured dispersion relationships agree well with the theories, indicating that the freesurface feature at the measurement location has a property related to gravity waves as well as turbulence.

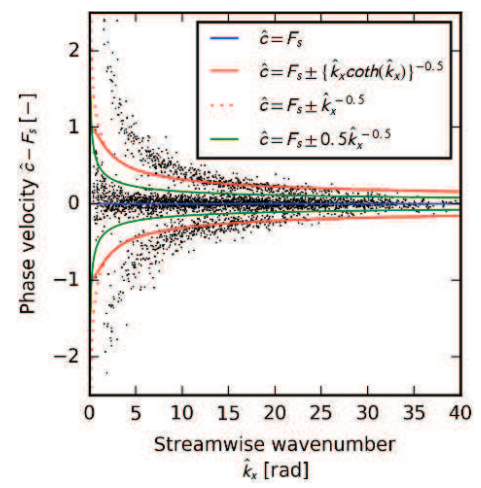

Fig. 6. Dispersion relationships extracted from STIs

\subsection{Effect of gravity waves on image-based flow measurement accuracy}

\subsubsection{Decompostion of wave and turbulence}

The unseeded image-based flow measurement technique such as STIV assumes that the freesurface fluctuations propagating as waves can be negligible and turbulence is much predominant. However, as shown in the previous section, the free-surface clearly exhibits both wave and of turbulence behaviour. Therefore, the effects by gravity waves need to be removed for improving the measurement accuracy. For efficiently removing the gravity wave components, regions related to turbulence and gravity waves are decomposed by the following relations, in which the separating boundaries are indicated by green curves in Figure 6. Each component can be picked up by masking the alternative region in the frequency domain.

(turbulence)

(wave)

$$
\begin{aligned}
& \hat{c} \leq F_{s} \pm 0.5 \hat{k}_{s}^{-0.5} \\
& \hat{c} \geq F_{s} \pm 0.5 \hat{k}_{s}^{-0.5}
\end{aligned}
$$

Figure 7 provides the standardised STIs composed of only the turbulence effect, with its original STIs shown in Figure 3. Obviously, the wavy patterns appears in the original STIs are eliminated and only the linear texture are efficiently extracted from the original STI. By applying these procedure to each array of geometric rectified video images, it is also possible to decompose the image into wave-related or turbulence-related textures directly from a geometric rectified video without creating STIs. Figure 8 shows the result of the decomposition performed directly from the geometric rectified video images shown in Figure 2(b). The target area is a square with a height of 400 pixels, $30.48 \mathrm{~m}$ in physical scale, the center of the image. It can be seen that the surface texture is advected to left without changing its general feature.

\subsubsection{Improvement of space-time image velocimetry}

In addition to the original STIV [2], a new algorithm called QESTA (Quality Evaluation of STI by using Two-dimensional Autocorrelation function) [7] is proposed, which can evaluate the accuracy of the estimated velocity in a form of error ratio. The error ratio is estimated 


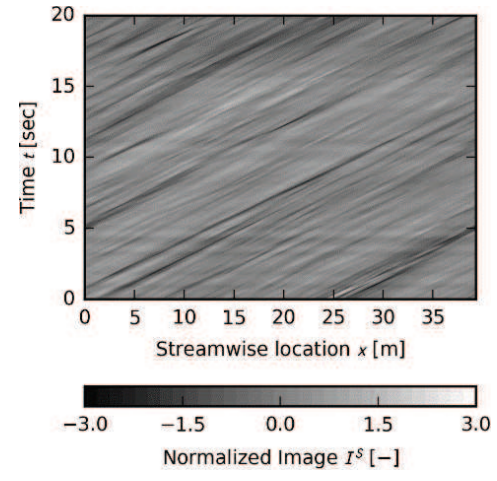

(a) $z=35.3 \mathrm{~m}, h=4.94 \mathrm{~m}$

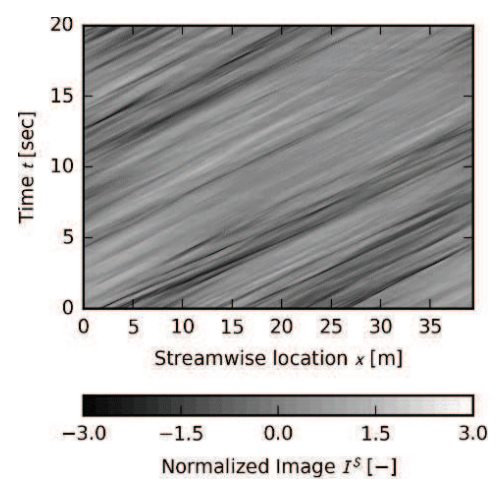

(b) $z=70.6 \mathrm{~m}, h=3.35 \mathrm{~m}$

Fig. 7. Standardized STIs of decomposed turbulence ( $z$; distance from the left bank)

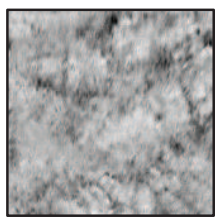

$\mathrm{t}=0.0[\mathrm{sec}]$

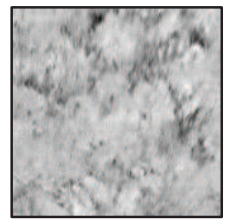

$\mathrm{t}=1.0[\mathrm{sec}]$

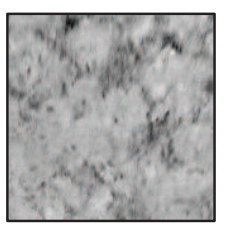

$\mathrm{t}=2.0[\mathrm{sec}]$

Fig. 8. Snap shots of decomposed turbulence directly from the video images

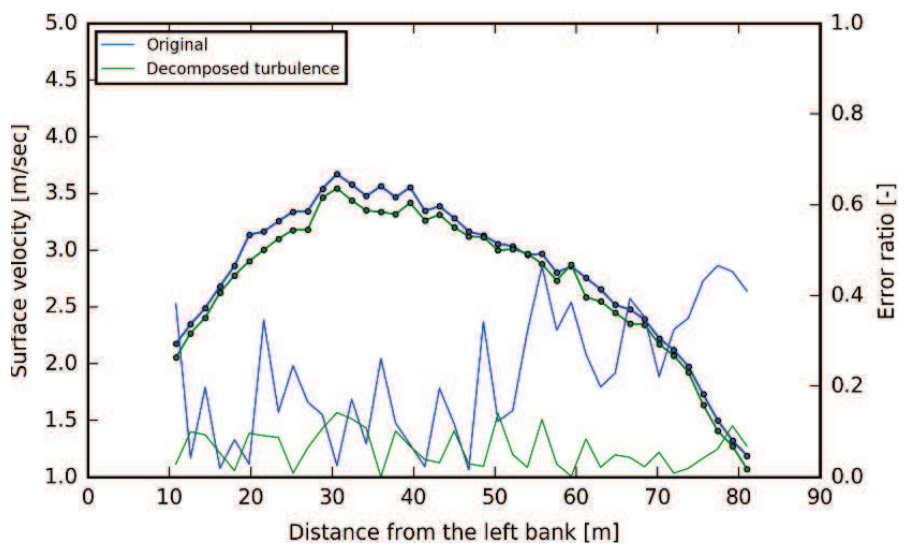

Fig. 9. Results of the STIV by using QESTA as an angle detection algorithm; the marks show the velocity and the solid lines show the error rate

from the shape of the two-dimensioanl autocorrelation function of the STI [7]. Figure 9 shows the velocity distributions measured by using the original STI and the STI including only the turbulence related textures such as shown in Figure 7. The error ratio for each case is indicated in the figure. As can be seen from Figure 9, advection speed of the turbulencerelated texture is a little slower than the speed using the original texture, suggesting that the generation of gravity wave accelerated the surface flow by a few percent. In addition, the error ratio decreases after decomposing the turbulence-related texture. On the other hand, the error ratio increases significantly closer to the other side of the river when the original texture is used. This might be due to the decrease of water depth in the other side, where the influence of the bottom roughness tends to generate relatively larger wave components. 


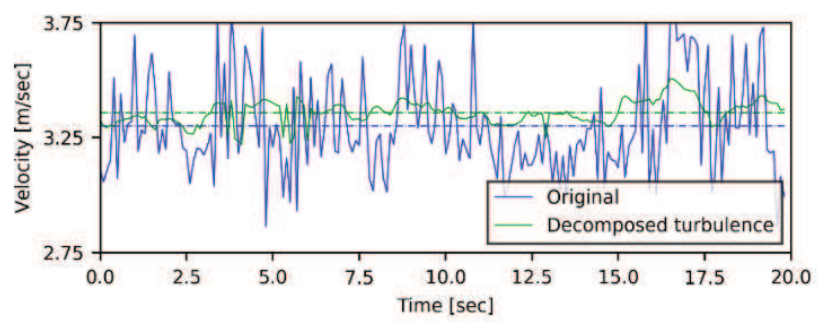

Fig. 10. Results of the LSPIV; the solid line shows the timeline of streamwise velocity and the dotted line indicates the mean velocity.

\subsubsection{Large scale particle image velocimetry}

In order to demonstrate the performance of extracting turbulence-related textures, the large scale particle image velocimetry (LSPIV) [1] was applied to the video images before and after the decomposition. The search window was set at the centre of the image and its size was set at 51 pixels. The measurement was performed by using 600 frames, equivalent to 20 seconds. Figure 10 shows the time variations of streamwise velocity component for each case. Although the mean value does not differ much with each other, the instantaneous velocity distribution is quite different, i.e. the amplitude of the original image shows much larger amplitude probably due to the wave effect. Therefore, extraction of turbulence-related texture by the proposed method could be used for improving the LSPIV measurement.

\section{Conclusions}

The wavenumber-frequency analysis was applied to space-time image textures used in STIV and turbulence-related textures that travel with the surface velocity were extracted efficiently for improving the measurement accuracy of STIV as well as LSPIV. The analysis also demonstrated that the free surface behaviour is subject to combined effects due to gravity waves and subsurface turbulence. The proposed method utilizing the wavenumber-frequency analysis could be used to improve the image-based measurement techniques significantly by removing the effect of gravity waves propagating in all directions on the water surface.

The measurement instrument using a high-accurate clinometer was prepared by TOKEN C.E.E. Consultants Co.Ltd. We are grateful to their support in the field measurement in the Shinano River.

\section{References}

1. I. Fujita, M. Muste, A. Kruger, J. Hydaulic Research, 36.3, 397 (1998)

2. I. Fujita, H. Watanabe, R. Tsubaki, Int. J. River Basin Man. 5, 105 (2007)

3. R. Savelsberg and W. Van de Water, J. Fluid Mech., 619, 95 (2009)

4. A. Nichols, S. Tait, K. Horoshenkov, J. Hydaulic Research, 54.4, 516 (2016)

5. G. Dolcetti, K. Horoshenkov, A. Krynkin, S. Tait, Physics of Fluid, 28, 105105 (2016)

6. K. Tani, I. Fujita, H. Yoshimura,Proceedings of the 37th IAHR W. C. (2017)

7. Y. Notoya, I. Fujita, S. Tateguchi, Annual J. Hydraulic Engineering, JSCE [in Japanese], 61, 505 (2017) 